This information is current as of April 26, 2023.

\title{
Temporal Association of Annular Tears and Nuclear Degeneration: Lessons from the Pediatric Population
}

A. Sharma, M.S. Parsons and T.K. Pilgram

AJNR Am J Neuroradiol 2009, 30 (8) 1541-1545

doi: https://doi.org/10.3174/ajnr.A1625

http://www.ajnr.org/content/30/8/1541 


\section{ORIGINAL RESEARCH}

\author{
A. Sharma \\ M.S. Parsons \\ T.K. Pilgram
}

\section{Temporal Association of Annular Tears and Nuclear Degeneration: Lessons from the Pediatric Population}

BACKGROUND AND PURPOSE: Studies done mainly in adults have shown an association between annular tears and nuclear degeneration. We wanted to study this association in the pediatric population to better understand the natural history of disk degeneration in its early stages. We hypothesized that this association is discernible even at a young age and that annular tears precede nuclear degeneration.

\begin{abstract}
MATERIALS AND METHODS: Twenty-six children with back pain and known disk pathology were identified from our radiology report data base. Two neuroradiologists independently evaluated T12 through S1 intervertebral disks in these images. One reader evaluated the disks for the presence and type of annular tears. The other reader graded the signal intensity of the disks on an ordinal scale and the extent of disk degeneration on the Pfirrmann scale. Mean degeneration and signal-intensity grades were compared for disks with radial tears, disks with nonradial tears, and disks without annular tears.

RESULTS: Fifty-six disks had radial tears. These demonstrated significantly higher nuclear degeneration grades and greater signal-intensity loss than disks with nonradial tears or disks with no annular tears. About one third (30.3\%) of the disks with radial tears had a normal nuclear signal intensity. Only $3 \%$ of disks with a signal-intensity grade of $\geq 3$ had an intact annulus.
\end{abstract}

CONCLUSIONS: Nuclear degeneration in children is associated with radial annular tears and rarely occurs in the absence of annular tears.
A nimal studies have demonstrated that artificially induced annular tears can result in subsequent nuclear degeneration. ${ }^{1}$ Whether naturally occurring annular tears play a similar role in the history of disk degeneration remains unknown. Previous studies, mainly in adults, have demonstrated a strong association between annular tears and nuclear degeneration ${ }^{2-4}$ and a faster nuclear degeneration in disks with annular tears. ${ }^{5}$ Intervertebral disks in the adult population have a high prevalence of both advanced nuclear degeneration and annular pathology, making it difficult to establish whether annular pathology precedes the nuclear degeneration or vice versa. A study focused on the pediatric population can be expected to provide a glimpse of the early stages of disk degeneration. The relative distribution of annular tears and nuclear degeneration in such a population can help us to better define the relative timing of the appearance of annular tears and nuclear degeneration.

Some excellent studies analyzing the process of disk degeneration have included some disk specimens from young patients. ${ }^{2,6-9}$ However, representation from the first 2 decades of life has been relatively small in these studies, ranging from 19 to 50 disks, ${ }^{6,9}$ possibly explaining a lack of direct statistical analysis focused on the young disks. Studies that do focus on the disk degeneration in children either have not included

Received February 23, 2009; accepted after revision March 18.

From the Mallinckrodt Institute of Radiology (A.S., M.S.P., T.K.P.), Washington University School of Medicine, St. Louis, Mo; Department of Radiology (A.S., M.S.P.), Barnes-Jewish Hospital South, St. Louis, Mo; and Department of Radiology (A.S., M.S.P.), St. Louis Children's Hospital, St. Louis, Mo.

Paper previously presented in part at: Annual Symposium of the American Society of Spine Radiology, June 19, 2009; Lake Buena Vista, Fla.

Please address correspondence to Aseem Sharma, MD, Mallinckrodt Institute of Radiology, Washington University School of Medicine, Campus Box 8131, 510 S Kingshighway Blvd, St. Louis, M0 63110; e-mail: sharmaa@mir.wustl.edu

DOI 10.3174/ajnr.A1625 annular tears in their analysis ${ }^{10,11}$ or have mainly looked at the clinical implications of annular tears. ${ }^{12}$

The purpose of our study was to look for the association of annular tears and nuclear degeneration in the pediatric population. We hypothesized that annular tears are associated with nuclear degeneration in this group. We also hypothesized that annular tears precede the appearance of nuclear degeneration. On the basis of this hypothesis, we expected to find that most disks showing nuclear degeneration also showed the presence of annular tears. By the same token, we expected to find nuclear degeneration common in disks with annular tears but not in those without.

\section{Materials and Methods}

This retrospective study was approved by the institutional review board of our hospital.

Patient Selection. Twenty-six children with reported findings of annular tear or disk herniation (protrusion or extrusion) on at least 1 level on prior MR imaging were selected from our radiology data base. The subjects included 8 males and 18 females. They ranged in age from 9 to 21 years (mean, $15.5+2.9$ years). None of the patients underwent diskography.

MR Imaging Analysis. The MR imaging was performed on 1.5T (Magnetom Sonata; Siemens, Erlangen, Germany) or 3T (Magnetom Trio; Siemens) scanners. All scans included sagittal and axial T2weighted turbo spin-echo images, which were used for the study. The section thicknesses for sagittal and axial images were 3 and $5 \mathrm{~mm}$, respectively. The axial images were obtained in the plane of the disk. TR ranged from 2090 to $7180 \mathrm{~ms}$, whereas the TE values ranged from 112 to $149 \mathrm{~ms}$. Two neuroradiologists read the studies independently. A total of 156 disks were examined. One reader evaluated the disks between the T12 and S1 segments for the presence of annular tears and their conspicuity and noted whether a given annular tear had characteristics of a radial tear. The second reader, in an independent evaluation, graded the disks for extent of loss of central signal inten- 

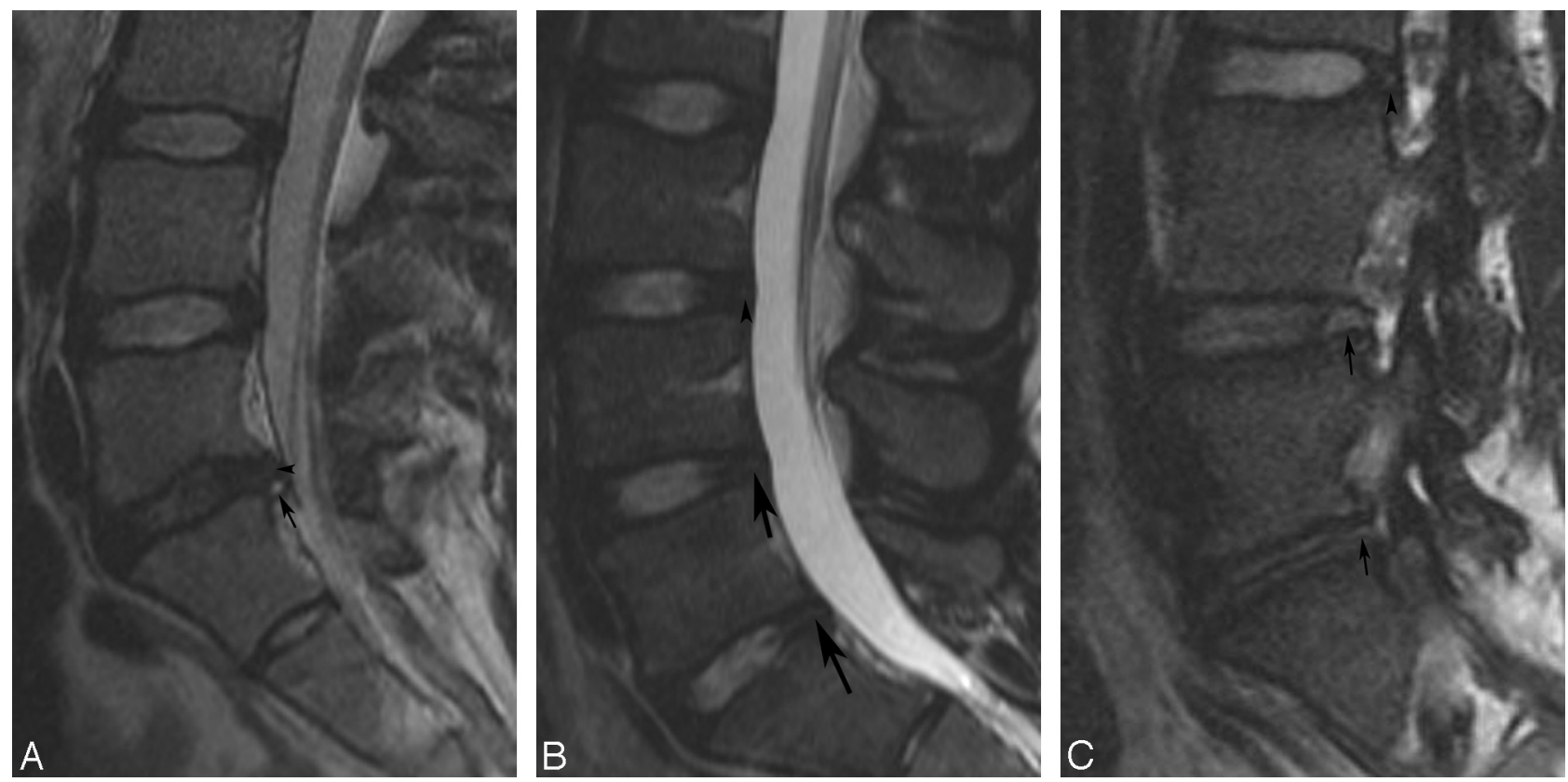

Fig 1. Examples of nuclear degeneration and annular tears. $A$, Sagittal T2-weighted image of the lumbar spine in a 16-year-old girl with a grade 1 annular tear at L5-S1 level (arrow). The tear is considered radial based on less conspicuous intermediate signal intensity extending across almost the entire thickness of the annulus (arrowhead). Nuclear degeneration is noted at this level with loss of signal intensity and minimal height loss. This disk was graded 4 on both signal-intensity and degeneration scales. Note the absence of annular tears and preserved signal intensity of the nucleus at L3-4 and L4-5 levels. B, Sagittal T2-weighted image of the lumbar spine from a 17-year-old boy with a grade 3 radial tear at $L 4-5$ (arrow), a grade 2 radial tear at L5-S1 (arrow), and a grade 3 nonradial tear (arrowhead) at the L3-4 level. Disks at all these levels were assigned a signal-intensity grade of 1 (L3-4 and L4-5) or 2 (L5-S1) and a degeneration grade of 2. C. Parasagittal T2-weighted image demonstrates radial (arrows) and nonradial (arrowhead) tears in the foraminal zones of the disks in an 18-year-old girl.

sity on T2-weighted images and also assigned a degeneration grade to the disk on the basis of a scale recommended by Pfirrmann et al. ${ }^{13}$ Definitions of the various parameters obtained are given below.

Six of the patients were chosen for assessing the intraobserver and interobserver variability of the imaging analysis. For this assessment, both readers performed a comprehensive evaluation of the annulus as well as the nucleus and then repeated these evaluations 10 days later.

Signal-Intensity Grade. A previously described ordinal scale was used to assess the extent of signal-intensity loss on T2-weighted images involving the central aspect of the disk, from grade 1 through $6 .{ }^{5}$ Disks were graded after assessing their entire extent on sagittal T2weighted turbo spin-echo images. Grade 1 was assigned to the disks demonstrating normal bright signal intensity in the central portion, whereas grade 6 implied complete absence of bright signal intensity in the central portion of the disk. Grades 2-5 represented loss of signal intensity affecting $<25 \%, 26 \%-50 \%, 51 \%-75 \%$, and $76 \%-99 \%$, respectively, of the central aspect of the disks.

Degeneration Grade. The extent of disk degeneration was also graded on a scale recommended by Pfirrmann et al. ${ }^{13}$ In addition to signal-intensity loss, this scale takes into account multiple other morphologic changes observed in the degenerated disks, such as blurring of the interface between the annulus and the nucleus pulposus and loss of height.

Annular Tear and Annular Tear Conspicuity. The presence of any hyperintense signal intensity within the peripheral annulus was considered to represent an annular tear (Fig 1). The conspicuity of the annular tears was graded on a scale ranging from 1 (signal intensity matching that of CSF) through 4 (presence of probable subtle intermediate signal intensity in the peripheral annulus) (Fig $1 A-C$ ). Grade 2 tears represented an obvious hyperintensity, though less intense than CSF. Grade 3 was assigned to tears showing definite but less conspicuous intermediate signal intensity in the peripheral annulus.
For tears varying in conspicuity along their extent, the grading was based on the most conspicuous region (Fig $1 A$ ). The entire circumference of the disk was evaluated to assess annular integrity.

Radial Tear. An annular tear was characterized as radial if abnormal signal intensity was seen extending from the innermost aspect of the annulus through almost the entire thickness of the annulus, at least into its outer third (Fig $1 A-C$ ).

Statistical Analysis. The intraobserver and interobserver variability was measured by calculating the $\kappa$ statistic. Mean signal-intensity grade and mean degeneration grades were compared for disks with and without annular tears, and the differences were tested for statistical significance with the Wilcoxon rank sum test. These analyses were performed for all disks collectively and for subgroups corresponding to upper (T12 through L3) and lower (L3 through S1) spinal segments. Mean signal intensity and degeneration grades were also calculated and compared for disks with radial tears, disks with nonradial tears, and disks with no annular tears. These differences were tested for statistical significance with the Kruskal-Wallis test. All statistical analysis was performed by using JMP 6.0 (SAS Institute, Cary, NC). A $P$ value $<.05$ was considered significant.

\section{Results}

\section{К Analysis}

There was substantial-to-almost-perfect intraobserver agreement for signal-intensity and degeneration grading, with $\kappa$ ranging from 0.77 to $0.87 .{ }^{14}$ Intraobserver agreement was almost perfect for 1 reader for annular tear identification and its characterization as radial $(\kappa, 0.93-1)$. Intraobserver agreement was fair to moderate for the other reader $(\kappa, 0.38-0.57)$. Interobserver agreement was fair to moderate for annular tear identification, radial tear identification, signal-intensity grad- 


\begin{tabular}{|c|c|c|c|c|c|c|}
\hline & \multicolumn{6}{|c|}{ Signal-Intensity Grade } \\
\hline & 1 & 2 & 3 & 4 & 5 & 6 \\
\hline Disks with no annular tears $(n=62)$ & $44(70.96 \%)$ & $16(25.81 \%)$ & $2(3.22 \%)$ & $0(0.00 \%)$ & $0(0.00 \%)$ & $0(0.00 \%)$ \\
\hline Disks with nonradial tears $(n=38)$ & $22(57.89 \%)$ & $10(26.31 \%)$ & $6(15.79 \%)$ & $0(0.00 \%)$ & $0(0.00 \%)$ & $0(0.0 \%)$ \\
\hline Disks with radial tears $(n=56)$ & $3(5.35 \%)$ & $14(25.00 \%$ & $11(19.64 \%)$ & $11(19.64 \%)$ & $15(26.78 \%)$ & $2(3.57 \%)$ \\
\hline Total $(N=156)$ & 69 & 40 & 19 & 11 & 15 & 2 \\
\hline
\end{tabular}

\begin{tabular}{|c|c|c|c|c|c|}
\hline & \multicolumn{5}{|c|}{ Degeneration Grade } \\
\hline & 1 & 2 & 3 & 4 & 5 \\
\hline Disks with no annular tears $(n=62)$ & $27(0.00 \%)$ & $32(79.45 \%)$ & $3(20.54 \%)$ & $0(0.00 \%)$ & $0(0.00 \%$ \\
\hline Disks with nonradial tears $(n=38)$ & $12(31.58 \%)$ & $22(57.89 \%)$ & $4(10.53 \%)$ & $0(0.00 \%)$ & $0(0.00 \%$ \\
\hline Disks with radial tears $(n=56)$ & $0(0.00 \%)$ & $14(25.00 \%)$ & $18(32.14 \%)$ & $21(37.50 \%)$ & $3(5.36 \%$ \\
\hline Total $(N=156)$ & 39 & 68 & 25 & 21 & 3 \\
\hline
\end{tabular}

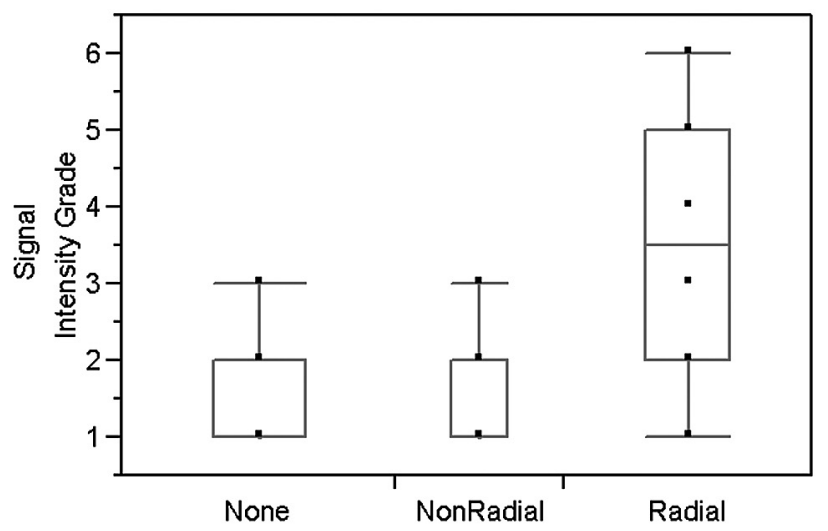

A

Tear Type

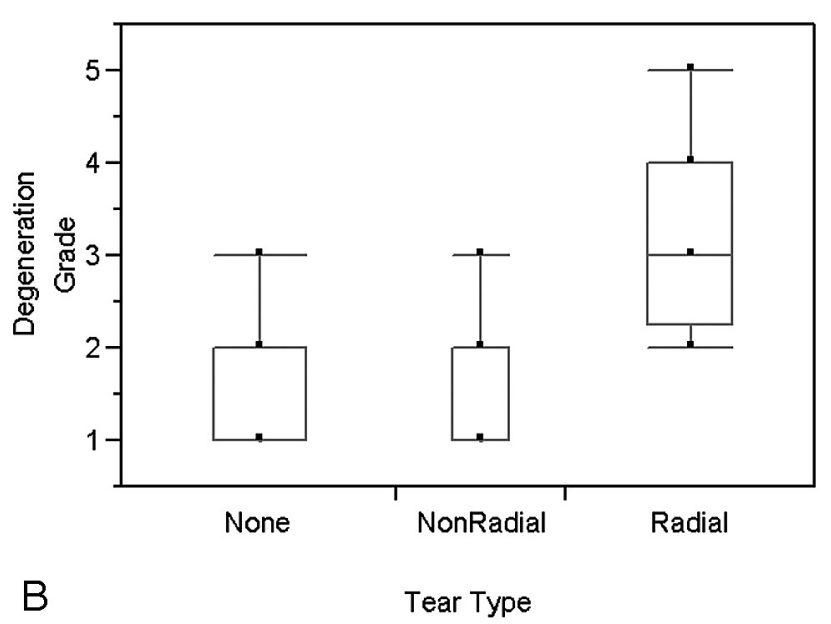

Fig 2. Boxplots of signal-intensity grades $(A)$ and degeneration grades $(B)$ of disks without annular tears, disks with nonradial tears, and disks with radial tears showing significantly higher signal-intensity and degeneration grades for disks with radial tears compared with the other 2 groups.

ing, and degeneration grading $(\kappa: 0.27,0.52,0.46$, and 0.39 , respectively).

\section{MR Imaging Assessment}

Ninety-four disks (60.2\%) had annular tears (Tables 1 and 2). Of these, 56 (59.5\%) were characterized as radial. The segmental distribution of the tears revealed an increase along more caudal segments of the spine for all tears in general and radial tears in particular. Most tears (45 disks, 47.8\%) were graded 3 on the conspicuity scale. Almost all of the remaining tears were classified as grade 2 (35 disks, 35. 2\%) or grade 1 (12 disks, $12.8 \%)$.

Of 62 disks without annular tears, 60 (96.7\%) demonstrated relatively preserved signal intensity, with signal-intensity grades of 1 and 2 (Table 1 ). There were only 2 disks (3.2\%) without annular tears that showed a signal-intensity grade of $\geq 3$. In contrast, 45 disks $(46.7 \%)$ with annular tears demonstrated a signal-intensity grade of $\geq 3$. This contrast was even more apparent when radial tears were considered in isolation. In this subgroup, 39 disks (69.6\%) demonstrated a signal-intensity grade of $\geq 3$ (Fig $1 A$ ). Seventeen disks with radial tears (30.3\%) demonstrated preserved signal intensity with a grade of 1 or 2 (Fig $1 B$ ). Similar trends were seen for the distribution of degeneration grades among disks without and with annular tears (Table 2).

Disks without annular tears demonstrated a mean signalintensity grade of $1.32 \pm 0.53$ and a degeneration grade of $1.61 \pm 0.58$. Mean signal-intensity and degeneration grades for disks with annular tears were $2.71 \pm 1.46$ and $2.64 \pm 1.06$, respectively. The differences were statistically significant $(P<$ .0001 , Fig 2). Disks with radial tears showed significantly higher signal-intensity and degeneration grades $(3.4 \pm 1.36$ and $3.2 \pm 0.89$, respectively) when compared with disks without annular tears or disks with nonradial tears $(P<.0001$, Fig $2)$. No significant differences were noted for signal-intensity and degeneration grades for disks with nonradial tears and disks without annular tears ( $P=.1$ and .16, respectively) (Fig 2).

\section{Discussion}

Our results demonstrate that a strong association of annular tears and nuclear degeneration is observed even in a pediatric patient population, an age group likely to reflect relatively early stages of the natural history of degenerative disk disease. Annular tears and nuclear degeneration were associated, even when the upper and lower segments of spine were evaluated separately, to minimize the possible confounding effects of 
axial loading. Although this association has been suspected on the basis of prior studies, ${ }^{3-5,9,15}$ it has not been clearly established in the pediatric population. On the basis of our prior experience with the adult spine, these results are not surprising. However, the present results suggest that significant changes in both the nucleus and the annulus appear soon after the process of disk degeneration is initiated (Fig 1A). The disks with annular tears demonstrated a mean degeneration grade of 3.2 on the Pfirrmann scale in our study. The presence of such advanced nuclear degeneration at a young age indicates that degenerative disk disease is a pathologic state distinct from aging. The similarity in the mean degeneration grade of these pediatric disks to the previously reported value of 3.1 in the adult population also suggests that the temporal course of nuclear degeneration in the pediatric and adult populations is probably similar, having more to do with the time of onset of the degenerative process rather than with the age of the patient. $^{5}$

Our results highlight the importance of radial tears in the process of disk degeneration, as has been stressed by previous authors. ${ }^{2,9,15}$ In our study, the appearance of nuclear degeneration was restricted mainly to the disks with radial tears. As noted above, no significant differences were found in signalintensity and degeneration grades for disks with nonradial tears and disks without annular tears. The role of nonradial tears in the process of disk degeneration remains unclear. Previous authors have discussed the mucinous degeneration or annular disorganization occurring in the annulus before the appearance of concentric annular tears. ${ }^{9,16}$ It is also possible that nonradial tears may weaken areas along the annulus, making it more prone to further injury and probable conversion to radial tears at a later date. More longitudinal studies will be needed to explore this further.

In our series, it was rare to see disks with a signal-intensity grade of $>2$ in the absence of concomitant annular tears. Annular tears were noted in 45/47 of such disks. However, patchy areas of signal-intensity loss in the nucleus, restricted to $<25 \%$ of its volume, could be seen in the absence of any annular tears. These results are similar to those of previous studies highlighting the differences in the histopathology of intervertebral disks of small children compared with those of adults. Yu et $\mathrm{al}^{2,15}$ and Ho et $\mathrm{al}^{7}$ have described an increasing fibrous content of the nucleus from "immature" disks in the first 2 years of life to the "adult" disks after maturation, with the appearance of a well-defined firm fibrous band along the equator of the disk. Similar changes in the nucleus have been reported by Haefali et al, ${ }^{9}$ who noted the presence of fibrous tissue peripherally in $38.4 \%$ of disks from individuals $11-30$ years of age.

The presence of such patchy areas of fibrous tissue probably accounts for most of the grade 2 disks in our series. It is likely that many of the small areas of loss of T2 signal intensity reflect maturation of the disk during early development in response to upright posture soon after infancy and to increasing demands of axial loading during childhood and adolescence. This process of maturation, however, does not produce more significant loss of signal intensity. In a study of the signal intensity of normal intervertebral disks, Sether et $\mathrm{al}^{8}$ showed that normal intervertebral disks demonstrate some, though mild, age-related decrease in signal intensity on T2-weighted images. Our results also indicate that though minimal nuclear signal-intensity loss may be seen normally, more pronounced loss of signal intensity mostly denotes a pathologic process, almost always occurring in the presence of radial annular tears.

Although a pronounced loss of signal intensity of the nucleus pulposus was almost always accompanied by annular tears in our series, the converse was not true. This distribution of signal intensity and degeneration grades supports our second hypothesis that annular tears appear earlier than the nuclear degeneration. Both radial and nonradial tears were found in disks with preserved signal intensity of the nucleus pulposus (Fig $1 B$ ). Disks with signal intensity and degeneration grades of $\leq 2$ accounted for $30.3 \%$ and $25 \%$ of all radial tears, respectively. Similar distribution of signal intensity and degeneration grades in disks with and without annular tears has been described in the adult population. ${ }^{5}$ These results are, however, discordant with the observations of some prior investigators. ${ }^{9,12}$ Haefeli et $\mathrm{al}^{9}$ found that radial tears were relatively rare in first 2 decades of life, and nuclear clefts preceded radial and concentric tears. ${ }^{9}$ There are a number of factors that may explain these differences. First of all, the disks in the 2 studies are from very different populations. Although all our patients had back pain or radiculopathy necessitating an MR imaging, disks in the study by Haefeli et al were from routine postmortem examinations of patients with no history of back pain. This certainly would explain a much higher prevalence of annular tears in our study. It is also possible that they missed some of the radial tears because they evaluated only midsagittal specimens for the age group in question.

Because we were evaluating the entire extent of the circumference of the disk, we were in a better position to notice radial tears in planes other than midline (Fig 1C). Despite this apparent discordance, a significant association between nuclear clefts and radiating annular tears was found in the study of Haefeli et al as well. ${ }^{9}$ They also found 10 disks from subjects who died after the first 2 decades in which the radial tears were seen in the absence of nuclear clefts. We found a higher prevalence of annular tears than nuclear degeneration in our study, which contrasts with the observations of Kjaer et al. ${ }^{12}$ The prevalence of signal-intensity loss reported by them was higher relative to that of annular tears. They reported almost $21.2 \%$ disks with abnormal signal intensity (defined as intermediate or hypointense signal intensity) but only $7.3 \%$ disks with annular tears (including $5.0 \%$ of disks with "high-intensity zones"). Although the exact definition used by Kjaer et al is not entirely clear from their article, we think that this apparent discordance may partly be due to the fact that all our scans were obtained on a $1.5 \mathrm{~T}$ or $3 \mathrm{~T}$ scanner in contrast to the $0.2 \mathrm{~T}$ scanner used in their study. Another contributing factor might be the fact that our definition of annular tears included all degrees of signal-intensity alteration within the annulus. As noted before, almost half of the annular tears in our study were graded 3 on the conspicuity scale, representing intermediate signal intensity within the annulus. Given that almost $70 \%$ of annular tears in the series of Kjaer et al were categorized as "high intensity zones," it is likely that they focused mainly on the annular tears that were more readily evident, probably similar to those graded 1 or 2 on our conspicuity scale.

A definite determination of the exact timing of annular tears and their temporal association with nuclear degeneration 
is challenging and will require a prospective long-term follow-up of normal disks, with relatively frequently repeated imaging. However, common occurrence of annular tears (both radial and nonradial) in disks with preserved signal intensity of the nucleus pulposus and paucity of disks with imaging-evident nuclear degeneration in the absence of annular tears suggest that most annular tears appear before the changes of nuclear degeneration manifest on MR imaging.

Our study has some limitations. In view of relative rarity of disk degeneration in children, we chose to include patients with at least 1 level of proven disk pathology. Due to this selection bias, the absolute prevalence of disk pathology in our population is certainly not reflective of the general population. We attempted to minimize reader bias by having different readers evaluate 2 main components of the hypothesis (ie, annular tears and nuclear degeneration). Although there is no diskographic proof, MR imaging has been shown to be a fairly sensitive and highly specific technique for the diagnosis of annular tears, including radial tears. ${ }^{17}$ Our $\kappa$ analysis demonstrated only fair interobserver agreement for annular tear identification and conspicuity. Corresponding values, however, were better for radial tears and assessment of nuclear signal intensity and degeneration.

\section{Conclusions}

Annular tears show a strong association with nuclear degeneration even in the pediatric population. This association is mainly based on radial tears. The distribution of signal-intensity and degeneration grades suggests that annular tears appear before the changes of nuclear degeneration become apparent on MR imaging.

\section{References}

1. Osti OL, Vernon-Roberts B, Fraser RD. 1990 Volvo Award in experimental studies: anulus tears and intervertebral disc degeneration-an experimental study using an animal model. Spine 1990;15:762-67

2. Yu S, Haughton VM, Sether LA, et al. Criteria for classifying normal and degenerated lumbar intervertebral disks. Radiology 1989;170:523-26

3. Milette PC, Fontaine S, Lepanto L, et al. Differentiating lumbar disc protrusions, disc bulges, and discs with normal contour but abnormal signal intensity: magnetic resonance imaging with discographic correlations. Spine 1999;24:44-53

4. Dullerud R, Johansen JG. CT-diskography in patients with sciatica: comparison with plain CT and MR imaging. Acta Radiol 1995;36:497-504

5. Sharma A, Pilgram T, Wippold FJ 2nd. Association between annular tears and disk degeneration: a longitudinal study. AJNR Am J Neuroradiol 2009;30:500-06

6. Vernon-Roberts B, Moore RJ, Fraser RD. The natural history of age-related disc degeneration: the pathology and sequelae of tears. Spine 2007;32:2797-804

7. Ho PS, Yu SW, Sether LA, et al. Progressive and regressive changes in the nucleus pulposus. Part I. The neonate. Radiology 1988;169:87-91

8. Sether LA, Yu S, Haughton VM, et al. Intervertebral disk: normal age-related changes in MR signal intensity. Radiology 1990;177:385-88

9. Haefeli M, Kalberer F, Saegesser D, et al. The course of macroscopic degeneration in the human lumbar intervertebral disc. Spine 2006;31:1522-31

10. Tertti MO, Salminen JJ, Paajanen HE, et al. Low-back pain and disk degeneration in children: a case-control MR imaging study. Radiology 1991;180:503-07

11. Erkintalo MO, Salminen JJ, Alanen AM, et al. Development of degenerative changes in the lumbar intervertebral disk: results of a prospective MR imaging study in adolescents with and without low-back pain. Radiology 1995;196:529-33

12. Kjaer P, Leboeuf-Yde C, Sorensen JS, et al. An epidemiologic study of MRI and low back pain in 13-year-old children. Spine 2005;30:798-806

13. Pfirrmann CW, Metzdorf A, Zanetti M, et al. Magnetic resonance classification of lumbar intervertebral disc degeneration. Spine 2001;26:1873-78

14. Crewson PE. Reader agreement studies. AJR Am J Roentgenol 2005;184: 1391-97

15. Yu SW, Haughton VM, Ho PS, et al. Progressive and regressive changes in the nucleus pulposus. Part II. The adult. Radiology 1988;169:93-97

16. Hirsch C, Schajowicz F. Studies on structural changes in the lumbar annulus fibrosus. Acta Orthop Scand 1953;22:184-231

17. Kakitsubata Y, Theodorou DJ, Theodorou SJ, et al. Magnetic resonance discography in cadavers: tears of the annulus fibrosus. Clin Orthop Relat Res 2003;228-40 